\title{
WHO BULLETIN
}

\section{HOSPITAL CAUSE-OF-DEATH}

STATISTICS: WHAT SHOULD WE MAKE OF THEM? 2014;92:3-3A.

\section{THE JOY OF FACTS AND FIGURES. 2013;91:904-905.}

Seleccioné estos dos artículos sobre estadísticas de los recientes números del boletín de la OMS.

En el primero de ellos, editorial del primer número del año 2014, se reportan algunos de los estudios sobre la calidad de los diagnósticos de muerte en aquellos decesos ocurridos dentro de los hospitales: infartos del miocardio clasificados como accidentes vasculares encefálicos (AVE), SIDA como septicemia, diabetes o EPOC codificadas como mal definidas. Errores que subestiman varias veces causas específicas de muerte. Las sugerencias nos dejan inquietos: no es un problema de entrenamiento o desconocimiento.

El segundo, una entrevista a Hans Rosling, que si bien señala algunos errores gruesos en el uso de estadís- ticas y las dificultades de un manejo experto inaccesible para los votantes, da algunas claves para recuperar el optimismo que el editorial de enero hubiera lesionado. No en vano Hans Rosling es cofundador de Gapminder Project (http://www. gapminder.org) y el director de The Joy of Stats, un documental que explora la importancia de las estadísticas. En esta entrevista, Rosling apunta no solo a la publicidad de las cifras, sino también a su construcción con significados, tanto desde el punto de vista de los decisores como para el público general.

Yuri Carvajal

Escuela de Salud Pública Facultad de Medicina Universidad de Chile 


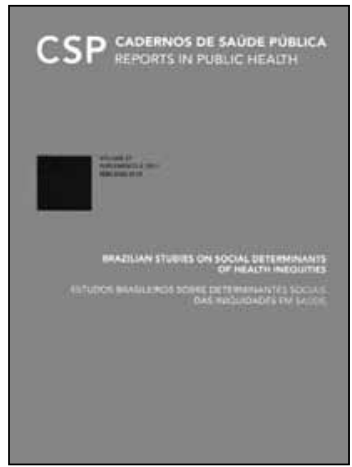

Recuerdo haber leído un artículo en que antropólogos gringos explicaban a las mujeres del altiplano boliviano la conveniencia de consumir agua previamente hervida, u otro en que Cuerpos de Paz proyectaban películas sobre las moscas y las infecciones a indígenas de la Amazonía brasileña. El desarrollo de las ciencias sociales era incipiente; algunas, como la sociología, recién estaban institucionalizándose; las corrientes que venían del norte eran dominantes sin lugar a duda. En todo caso, experiencias como las anotadas formaban parte del verdadero inicio de la intervención de las ciencias sociales en medicina.

Estamos hablando de finales de los años sesenta. Varios aspectos incidieron para que este panorama fuera cambiando. Entre ellos, la participación de Juan César García, sus trabajos y actividades, sobre todo si lo consideramos desde un punto de vista continental. Y esta circunstancia justifica sobradamente una 'revisita' a su obra, trayectoria e impacto. Everardo Duarte Nunes, el autor de este artículo, tiene también sobrados derechos para esta incursión. Autor de varias recopilaciones de textos de Juan César García (por ejemplo, Juan César García: pensamiento social en salud, OPS/Interamericana, 1991), en este hace una aproximación a su sociobiografía destacando sus logros en las distintas etapas de su desempeño (1961-1971 y 19721984, año de su muerte).

Los primeros trabajos de Juan César García se centraban en temáticas propias de la sociología médica norteamericana, como era obvio en este tiempo. Incorporado al Departamento de Recursos Humanos de la OPS desde 1966, García desarrolla una valiosísima actividad de formación de recursos humanos en ciencias sociales y salud en América Latina, a través de la difusión de literatura pertinente y de la realización de reuniones de discusión y análisis que tienen lugar en distintos lugares de América. En este sentido, se destaca con mucho como articulador de grupos e instituciones. Esta actividad, en sinergia con los procesos sociales y políticos del continente, conduce a una particular "superación del biologismo y del funcionalismo entonces hegemónicos" en las ciencias sociales en salud. 
Coincidentemente con los vientos de la época, se recurre al estructuralismo marxista, lo que marcará la producción intelectual de Juan César García en adelante. En este sentido, y por su insistencia en el recurso a este vocabulario, se destaca en ese entonces como pensador innovador y consistente. Lo que siguió a partir de ahí es historia conocida. Aparecen muy diversos artículos, muchos de ellos en Educación Médica y Salud. En 1972 presenta en el Congreso Internacional de Sociología, desarrollado en Caracas, su trabajo "Las ciencias sociales en medicina”. Ese mismo año publica La educación médica en América Latina, su investigación de mayor envergadura (415 pp). Aparecen luego cursos de posgrado en medicina social (México 1973 y Brasil 1974), hay desarrollos institucionales y publicaciones importantes en Brasil, Ecuador, México, Argentina y Venezuela, reuniones en Cuenca (1972 y 1984), Río de Janeiro (1972) y otras. Juan César García fallece en 1984, y ese mismo año se crea ALAMES (Asociación Latinoamericana de Medicina Social).

Hasta aquí llega Nunes. De ahí para adelante todo es casi miel sobre hojuelas: la
OMS crea la Comision de los Determinantes Sociales en Salud en el 2005; viene Michael Marmot, informe del 2008 y los DSS se instalan casi definitivamente como una nueva epidemiología. Pero ALAMES obviamente se inquieta: lo que había comenzado como una visión contracultural de la salud pública se ha transformado, o anda cerca de transformase, en un nuevo pensamiento oficial.

Jorge Gaete Avaria Editor Asociado Revista Chilena de Salud Pública

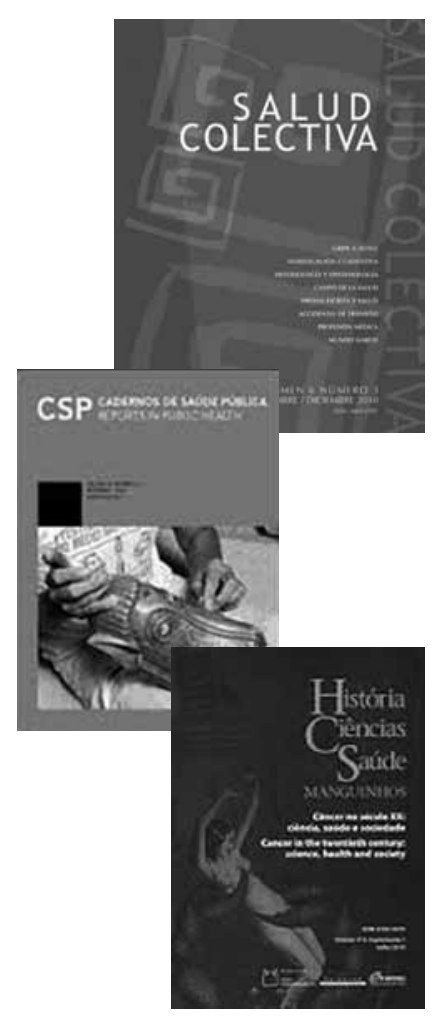

\section{REVISTA DE REVISTAS EN SALUD PÚBLICA: SALUD COLECTIVA; CADERNOS DE SAÚDE PÚBLICA; HISTÓRIA, CIÊNCIA, SAÚDE - MANGUINHOS.}

Como conocimiento, la salud pública es algo que se hace constantemente. La publicación de libros y revistas es parte de ese hacer. Los primeros suponen una visión más consolidada, una perspectiva con mayor nivel de condensación; las revistas, en cambio, la puesta en circulación de datos de la investigación en curso o la inmediatez de la reacción reflexiva ante hechos, circunstancias o ideas en discusión. Pero ambos productos participan de una misma actividad: poner en circulación ideas, aproximaciones, criterios. De compartir con otros y con todos; en suma, de hacerlo público. Y ahí se encuentran autores $\mathrm{y}$ escritores. Ambos desde sus propias perspectivas colaboran en la construcción de una opinión que está en la base del saber de la salud pública. Porque esta es la gracia del hacer público: puestas en circulación, las ideas irrumpen 
en la realidad, son agregadas a otras ideas, son combinadas con ellas, son puestas en competencia. Y así se va construyendo el conocimiento...

Con estos criterios en mente he revisado los últimos números de tres revistas emblemáticas, al decir de hoy, dentro del campo: Salud Colectiva, Cadernos de Saúde Pública e História, Ciências, Saúde-Manguinhos.

La primera es una publicación científica cuatrimestral editada por el Instituto de Salud Colectiva de la Universidad Nacional de Lanús, cuyo objetivo es difundir artículos que contribuyan al estudio del proceso salud-enfermedad-atención-cuidado. El número 2 (abril-agosto de 2013) tiene como eje la compleja ecuación entre trabajo, economía y salud entre los trabajadores. Contiene un editorial de Carlos Aníbal Rodríguez sobre ciencia y ética en la salud de los trabajadores y tres trabajos sobre situaciones específicas. Entre ellos, uno titulado " ¿Salud y productividad?: sobre la formación de una analítica 'económica' de la relación salud-trabajo (Argentina, 19001955)", de Victoria Haidar, que incluye una referencia a la relación entre René Sand y Alejandro del Río. El número actual (3, septiembrediciembre de 2013) incluye un artículo del sociólogo José Buschini relativo a las formas que asume el desarrollo científico ("Renovación institucional y modernización científica: la creación del Instituto de Investigaciones He- matológicas a mediados de la década de 1950"). En este trabajo se presta particular atención a la conformación de las bases materiales y los mecanismos institucionales y culturales para el desarrollo de investigaciones científicas y de prácticas clínicas, asistidas por procedimientos y técnicas anclados en las ciencias básicas, así como también su relación con colectivos disciplinares cosmopolitas que les proveerán de temas, conceptos y procedimientos. Viene también un artículo de Mónica Serena Perner ("Transformaciones en el abordaje de la diabetes: análisis de las evidencias científicas publicadas por dos sociedades científicas (19802010)"). A través de un análisis de contenido de artículos, guías y consensos publicados por la Sociedad Argentina de Diabetes y la Asociación Latinoamericana de Diabetes, se analizan los cambios y modificaciones encontrados en las clasificaciones, en los valores considerados normales para definir a una persona como diabética, y en los respectivos tratamientos. Resuenan en este texto las críticas de Luis David Castiel a la hiperpreventización.

Cadernos de Saúde Pública, la segunda revista revisada, es una publicación de la Escola Nacional de Saúde Pública Sergio Arouca - Fundação Oswaldo Cruz, cuyo objetivo es publicar artículos originales que contribuyan al estudio de la salud pública y disciplinas relacionadas. Se trata de una voluminosa pu- blicación de 222 páginas, que en su número de noviembre de 2013 incluye dieciséis artículos de ciencia 'normal', para utilizar un término relativamente consagrado. Por ejemplo, "La aparición de dolor de espalda y los factores asociados en niños $\mathrm{y}$ adolescentes en una escuela privada en el sur de Brasil", "Factores asociados al tiempo libre y la candidiasis oral en niños que viven con VIH/ SIDA, São Paulo, Brasil”, "Prevalencia y factores asociados con la inactividad física en adolescentes de escuelas públicas en Uruguaiana, Rio Grande do Sul, Brasil” y otros similares. Con razón sus editoras se quejan en un editorial titulado “¿Más de la misma epidemiología?”: “A pesar del inmenso cuidado en la planificación, recogida y procesamiento de datos, la contribución de estos estudios es limitada, tanto en el avance del conocimiento científico, como en el impacto social de sus resultados".

El número siguiente de esta publicación, el correspondiente al mes de diciembre, parece responder mejor a lo que esperan las editoras. Comienza con un artículo acerca de "Los ensayos clínicos y la industria farmacéutica", vinculando entonces la aparición de distintas drogas en el mercado con las consideraciones económicas de la industria. Le sigue otro artículo acerca de "La salud y la vida buena: aportes del enfoque de las capacidades de Amartya Sen para el razonamiento ético en salud 
pública". Otros títulos son: "La violencia de género y los embarazos no deseados: prevalencia y factores asociados", "Estudio comparativo de las representaciones sociales sobre diabetes mellitus y pie diabético", "Equivalencia semántica y conceptual de la versión brasileña de Neighborhood Environment Walkability Scale for Youth (NEWS-Y)", "Medicina basada en evidencia en contexto; perspectivas desde una ciencia social crítica”.

La tercera revista revisada es Historia, Ciéncia, SaúdeManguinhos. Esta publicación presenta artículos que generalmente van más allá de la ciencia 'normal'. El índice del número julio/septiembre de 2013 desde ya es indicativo: para muestra mencionemos, por ejemplo, un artículo de Sandra Caponi acerca de "Quetelet, el hombre medio y el saber médico", otro de
José Augusto Leandro acerca de "A favor del sacrificio del aislamiento: lepra y filantropía en Argentina y Brasil, 1930-1946", y uno de Ana Cecilia Weintraub, "Contribuciones del pensamiento de Didier Fassin al análisis crítico de las políticas de salud dirigidas a poblaciones vulnerables". El número siguiente -primer suplemento del año- fechado en noviembre del 2013, está dedicado prácticamente entero a los estudios de ciencia, tecnología y sociedad (CTS): desde estudios que analizan cómo distintas publicaciones construyen una imagen social de la ciencia ("Leer ciencia en el Brasil del siglo XIX: Revista Popular 1859-1862”), de la tecnología y de la salud ("Las opiniones de los brasileños sobre ciencia y tecnología: la paradoja de la relación entre información y actitudes”), hasta las controversias cientí- ficas, incluyendo discusiones históricas, "No intento crear una polémica: la llegada de la peste al Brasil, análisis de una controversia, 1899", hasta otras más contemporáneas: "Fuera del orden natural: la naturaleza de los discursos acerca de la clonación y la investigación sobre células madres en los periódicos brasileños".

Conclusión: La salud pública que se elabora a través de las publicaciones indica la plena vigencia del paradigma epidemiológico convencional. Hay atisbos de otros desarrollos, fundamentalmente a través de los estudios de CTS, pero estos al parecer no se plantean como competencia para la epidemiología.

Jorge Gaete Avaria Editor Asociado Revista Chilena de Salud Pública 